must be carefully controlled to reduce the electric field within, so that electrostatic fields do not remove the ion from the trap and to avoid excess instability as the radiofrequency voltage is ramped down. An inner direct-current electrode was used to fine tune the field, reducing the residual electrostatic force on the ion to about $10^{-20} \mathrm{~N}$; this displaces the ion within the dipole trap but is less than the maximum trapping force, so the ion remains within the trap.

As the authors note, this is only a first demonstration of all-optical trapping of ions; many improvements are possible, and are necessary for the method to be useful in practice. The trapping lifetime that they achieved was only about $2.4 \mathrm{~ms}$, and the heating rate within the trap was $7.5 \mathrm{nK} \mathrm{ms}^{-1}$. Both of these can be improved by larger detuning of the laser wavelength, which will reduce the scattering rate as the trapping wavelength differs more from the wavelength of the spectral line used for trapping. However, as detuning will reduce the real part of the polarizability of the ion as well as the absorptivity (the complex part of the polarizability), a higherpowered laser will be required to achieve the same optical force. It should also be possible to use established methods for cooling trapped neutral atoms to improve the lifetime. The ability to do experiments using the interaction of multiple ions trapped, for example, in an optical lattice remains to be demonstrated. One approach might be to make use of hybrid traps, using both optical and radiofrequency traps together.

The development of all-optical ion traps involves an interesting interplay between necessity and possibility. Radiofrequency and static electromagnetic traps have long been the tools of choice for trapping ions. Their very operation depends on the strong coupling between fields and ions due to the ions' electric charge. The lack of this strong coupling meant that alternative methods had to be developed to trap neutral atoms, leading to the development of methods such as the alloptical dipole trap. The strong coupling that made radiofrequency and static traps possible for ions prevented the use of such all-optical traps for ions. However, meeting the experimental challenges posed by stray fields allows the fundamental limitations of radiofrequency traps to be overcome. A major limitation has been the radiofrequency-driven motion within such traps, which has so far prevented the achievement of low collision energies. The work of Schaetz and co-workers ${ }^{8}$ is a tangible first step towards the merging of what have until now been two very different experimental fields, atom trapping and ion trapping. Now we will be able to see what the combination of the strengths of the two fields will bring.

Timo Nieminen is in the School of Mathematics and Physics at The University of Queensland, Brisbane,

Queensland 4072, Australia.

e-mail: timo@physics.uq.edu.au

\footnotetext{
References

1. Chu, S. Rev. Mod. Phys. 70, 685-706 (1998).

2. Cohen-Tannoudji, C. N. Rev. Mod. Phys. 70, 707-719 (1998)

3. Phillips, W. D. Rev. Mod. Phys. 70, 721-741 (1998).

4. Moffitt, J. R., Chemla, Y. R., Smith, S. B. \& Bustamante, C. Annu. Rev. Biochem. 77, 205-228 (2008).

5. Barrett, M. D., Sauer, J. A. \& Chapman, M. S. Phys. Rev. Lett. 87, 010404 (2001)

6. Ashkin, A. Optical Trapping and Manipulation of Neutral Particles Using Lasers: A Reprint Volume with Commentaries (World Scientific, 2006)

7. Ashkin, A. IEEE J. Sel. Top. Quant. Electron. 6, 841-855 (2000)

8. Schneider, Ch., Enderlein, M., Huber, T. \& Schaetz, T. Nature Photon. 4, 772-775 (2010).
}

\title{
LIGHT-EMITTING DIODES
}

\section{Natural white light}

White light-emitting diodes (LEDs) have tremendous potential for energy-efficient illumination. However, achieving white light emission with a 'colour temperature' that is not too blue is a serious challenge.

$\mathrm{In}_{x} \mathrm{Ga}_{1-x} \mathrm{~N}$ compound semiconductor alloys are currently considered to be one of the most promising material systems for white LEDs, as they offer a broad direct bandgap that varies from the near-infrared $(0.6 \mathrm{eV}, \ln N)$ to the near-ultraviolet (3.4 eV, GaN). In principle this allows high-quality white LEDs to be realized by mixing $\ln _{x} \mathrm{Ga}_{1-x} \mathrm{~N}$ devices emitting at blue, green and red wavelengths. The problem is the dramatic drop in emission efficiency at longer (green and red) wavelengths. Because of the large lattice mismatch between InN and $\mathrm{GaN}(\sim 11 \%)$ and the polar nature of their crystal structures, long-wavelength devices based on high-In-content $\mathrm{In}_{x} \mathrm{Ga}_{1_{-x}} \mathrm{~N} / \mathrm{GaN}$ quantum wells have an unavoidably high density of defects and huge internal piezoelectric fields

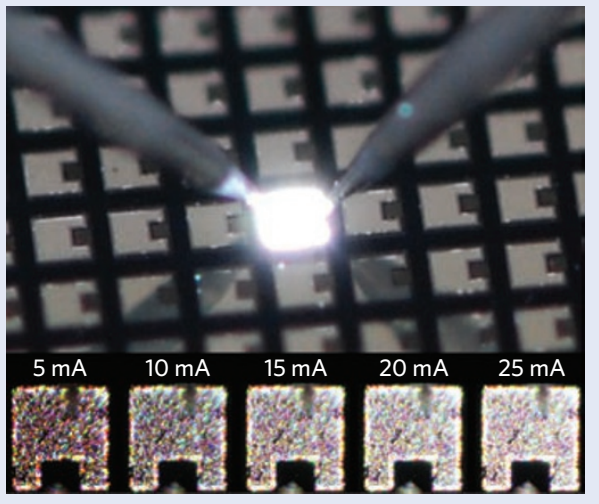

success relies on the use of a selfassembled nanorod geometry to eliminate the material strain. The team fabricated an array of $\ln _{x} \mathrm{Ga}_{1-x} \mathrm{~N} / \mathrm{GaN}$ nanorod heterostructures by plasma-assisted molecular beam epitaxy. Emission of natural white light was realized by designing each $\mathrm{GaN}$ nanorod $\mathrm{p}-\mathrm{n}$ junction to contain a stack of several nanodisk In GaN emitters, each emitting a different colour due to their varying thickness and composition.

At small drive currents, the observed electroluminescence was 'spotty' because only a small number of nanorods were electrically injected by the p-type contact pad (see image). However, the integrated electroluminescence intensity was found to increase linearly as a function of injection current, eventually becoming a uniform natural white (a colour temperature of $6,000 \mathrm{~K}$ ) at drive currents of $20 \mathrm{~mA}$ and above.

NORIAKI HORIUCHI 